\title{
ASPECTOS BIOLÓGICOS PESQUEROS DE Potamorhina altamazonica llambina (COPE, 1878) EN LA REGIÓN LORETO-AM AZONÍA PERUANA
}

\author{
Aurea GARCÍA ${ }^{1}$, Gladys VARGAS ${ }^{1}$, Ronald RODRÍGUEZ ${ }^{2}$, Víctor MONTREUIL ${ }^{3}$, Rosa ISMIÑO ${ }^{1}$, \\ Homero SANCHEZ ${ }^{1,3}$, Salvador TELLO ${ }^{1}$, Fabrice DUNPOCHELLE ${ }^{4}$ \\ 1 Instituto de Investigaciones de la Amazonía Peruana - IIAP. Programa para el Uso y Conservación del Agua y sus Recursos - \\ AQUAREC. \\ 2 Gobierno Regional de Loreto, Programa de Conservación, Gestión y Uso Sostenible de la Diversidad Biológica de Loreto - \\ PROCREL. \\ 3 Facultad de Ciencias Biológicas. Universidad Nacional de la Amazonía Peruana - UNAP. \\ 4 Institut de Recherche pour le Développement (IRD)-UM R-ISEM (Institut des Sciences de l'Evolution de M ontpellier)
}

\section{RESUMEN}

Información sobre los desembarques totales de Potamorhina altamazonica, muestran que estas se incrementan a través de los años, variando de 797 a 2,823 toneladas entre los años 1984 a 2009. La reproducción de la especie ocurre dentro del período comprendido entre noviembre a marzo, durante el período de incremento del nivel de las aguas del río. Esta especie alcanza la talla de primera madurez sexual $\left(\mathrm{L}_{50}\right)$ a los $16,3 \mathrm{~cm}$ de longitud estándar en hembras y 15,5 cm en machos. El análisis de la distribución de frecuencia de tallas muestra variaciones entre los porcentajes de ejemplares maduros e inmaduros entre un año a otro.

PALABRAS CLAVES: Potamorhina altamazonica, madurez sexual, época de reproducción, talla de primera madurez, Amazonía peruana.

\section{BIOLOGICAL ASPECTS OF FISHING Potamorhina altamazonica llambina (COPE, 1878) IN THE PERUVIAN AM AZON REGION-LORETO}

\begin{abstract}
Information on total landings of Potamorhina altamazonica, show that these are increasing through the years, ranging from 797 to 2,823 tons from 1984 to 2009. The reproduction of the species occurs within the period from November to march, which corresponds to the flooding season. The mean size at first sexual maturity (L50) is 16,3 $\mathrm{cm}$ standard length and $15,5 \mathrm{~cm}$ in females and males, respectively. The analysis of length frequency distribution shows variations in the proportions of mature and immature in the catches.
\end{abstract}

KEYW ORDS: Potamorhina altamazonica, sexual maturity, spawning season, size at maturity, Peruvian Amazon. 


\section{INTRODUCCIÓN}

Los desembarques pesqueros en la región Loreto esta relacionada con la composición trófica de las capturas. En Loreto las proporciones de piscívoros es relativamente baja y los desembarques están dominados por consumidores primarios (herbívoros y detritivoros), lo cual refleja la intensidad de pesca a la que son sometidas las especies, donde a medida que se incrementa la pesca de grandes especies, generalmente piscívoros, desapareces y son remplazados por especies más pequeñas pertenecientes al orden de los Characiformes quienes dominan los desembarques pesqueros durante las dos últimas décadas (García et al., 2009). Potamorhina altamazonica llambina, se ubica dentro de este grupo, y es la segunda especie más importante en las capturas en relación a las 65 especies desembarcadas. Su captura representa en promedio el $14 \%$ de los desembarques totales registrados en Loreto (García et al., 2009).

Potamorhina altamazonica, especie perteneciente a la familia Curimatidae se encuentra distribuida en las cuencas del río Amazonas y Orinoco (Vari, 1984). Se caracteriza por presentar migraciones estaciónales asociadas con la reproducción y alimentación (Smith, 1979). Esta especie presenta régimen alimenticio detritivoro, utiliza materia orgánica y microorganismos asociados al fondo de los lagos y márgenes de los ríos (Goulding, 1979). Sus larvas son comúnmente encontradas en el canal central del río Amazonas (Nascimento \& Araujo-Lima, 1993).

A pesar de su importancia en los desembarques de las últimas décadas, aun, son escasos los estudios básicos acerca de la biología de Potamorhina altamazonica. Por lo que existe una urgente necesidad de generar conocimientos previos que sirvan de base para la implementación de medidas eficientes para la conservación y uso responsable de la especie en su medio natural. Bajo este contexto, se diseñó este trabajo con el objetivo de determinar los principales parámetros del ciclo de la vida de la llambina.

\section{ATERIALY MÉTODOS}

Los datos fueron obtenidos de las capturas desembarcadas por la flota pesquera comercial y de las embarcaciones comerciales de carga y pasajeros en la ciudad de Iquitos, además de las pescas experimentales realizadas con pescadores de la localidad de Requena.

Se analizo información colectada entre 1996, 1997, 2005 a 2010. Además, de información de desembarque pesquero proporcionado por PRODUCE-LORETO, correspondientes al período 1984 a 2010.

\section{COLECTA Y M UESTREO DE M ATERIAL BIOLÓGICO}

Se registró datos biométricos como longitud total, horquilla y estándar con la ayuda de un ictiometro (graduado al milímetro), el peso fue registrado con una balanza de 0,1 gramos de precisión. Además de datos biológicos como sexo y estado de maduración de las gónadas.

\section{MÉTODOS PARA ELANÁLISIS DEDATOS}

Los parámetros reproductivos están basados en el análisis de un ciclo biológico.

La época de reproducción fue determinada mediante el análisis de la frecuencia mensual de ejemplares sexualmente maduros (Duponchelle et al., 1999, Nuñez \& Duponchelle, 2009). La escala de maduración gonadal fue elaborada mediante el análisis macroscópico de las gónadas, tomando como referencia la escala utilizada en García et al. (1996).

El factor de condición somático fue determinado, de acuerdo a la ecuación $\mathrm{K}=\mathrm{Wc} / \mathrm{L}_{\mathrm{t}}^{\mathrm{b}}$ donde $\mathrm{Wc}=$ peso corporal; $\mathrm{b}=$ coeficiente de regresión de la relación $\mathrm{W}_{\mathrm{t}} / \mathrm{L}_{\mathrm{h}}($ Vazzoler, 1996).

Los parámetros de la relación $\mathrm{W}=\mathrm{a} \cdot \mathrm{L}^{\mathrm{b}}$ fueron estimados después de la transformación logarítmica de los datos.

La longitud de primera madurez sexual, se determinó ajustando la proporción de individuos maduros (\%MF); a partir del estadio dos (2) de la escala de maduración, durante la época de reproducción, a intervalos de $1 \mathrm{~cm}$ de longitud estándar a una función logística, utilizando una regresión no linear, ponderado por el número total de individuos en cada clase de longitud (Duponchelle \& Panfili, 1998), utilizando la siguiente fórmula: $\% \mathrm{MF}=1 *(1+e(-$ $\left.\left.\mathrm{a}^{*}\left(\mathrm{~L}-\mathrm{L}_{50}\right)\right)\right)-1$. Donde: $\% \mathrm{MF}=$ Porcentaje de individuos maduros por clase de longitud; $\mathrm{L}=$ Valor central de cada clase de longitud; L50 = Valores constantes del modelo.

El estado de explotación de la especie fue estudiado usando indicadores de sobrepesca que recientemente se han propuesto como alternativas a modelos convencionales de evaluación: la proporción de ejemplares maduros en las capturas y la proporción de peces capturados a la longitud óptima $\left(\mathrm{L}_{\text {opt }}\right)$ (Froese, 2004). La proporción de individuos maduros en las capturas fue calculada como el porcentaje de individuos que alcanzan un tamaño mayor o igual a $\mathrm{L}_{50}$. El $\mathrm{L}_{\text {opt }}$ fue calculado como: $\log \mathrm{L}_{\text {opt }}=\mathrm{a} * \log \left(\mathrm{L}_{50}\right)-\mathrm{b}$ (Froese \& Binohlan, 2000), donde $\mathrm{L}_{50}$ es la longitud a la primera madurez sexual, a y b son constantes determinada para amplio rango de especies de peces $(a=1.053, b=0.0565)$. (Froese, 2004). 


\section{RESULTADOS Y DISCUSIÓN}

\section{ESTADÍSTICAS DE DESEM BARQUES}

Las capturas anuales de Potamorhina altamazonica llambina, en la región Loreto, incluyen especimenes fresco, salpreso y secosalado, variando de 797 a 2,823 toneladas entre los años 1984 a 2010 (Figura 1). Se observa una tendencia irregular de los desembarques con un incremento promedio a través de los 27 años $\left(\mathrm{y}=30.0818 x-58357, R^{2}=0.1706, p=0.0320\right)$. Los mayores desembarques se observa en la década de los 90, con capturas que superaran las 2,800 toneladas en los años 1992 y 1993; siendo ligeramente menor en la última década con el mayor registró en el 2004 y 2005.

\section{ÉPOCA DE REPRODUCCIÓN}

El inicio de la época de reproducción de Potamorhina altamazonica empieza en el mes de noviembre y finaliza en el mes de abril del año siguiente. Se observa un pico máximo de reproducción que va desde finales de un año (diciembre) al inicio de otro (febrero). Evento que ocurre en sincronización con el inicio del aumento del nivel de las aguas fase del ciclo hidrológico conocido como repiquete (Figura 2). Este comportamiento es característico de las especies amazónicas (Goulding, 1980; Lowe-McConnell, 1987; Tello et al., 1992; Ruffino \& Isaac, 1995; Isaac et al., 2000), y es considerado como estrategia para garantizar la sobrevivencia de un mayor número de descendientes, debido al suministro de alimento en áreas recientemente inundadas, asegurando la preservación de la especie al reducir su vulnerabilidad (Lowe-McConnell, 1987; Villacorta, 1997; Lauzanne etal., 1990).

\section{FACTOR DE CONDICIÓN SOM ÁTICO}

Se observó que los menores índices de condición somáticos (diciembre a febrero) coinciden con la máxima frecuencia de reproducción y el inicio de aumento del nivel de las aguas (Figura 3). Período en el cual la especie deja de alimentarse consumiendo parte de sus reservas energéticas acumuladas en las vísceras, músculos e hígado que es utilizado en el desarrollo de las gónadas y culmina en el desove (Vazzoler, 1996; Campos, 2005). Además se observa que la condición somática se incrementa considerablemente conforme las aguas alcanzan su máximo nivel después de ocurrido el desove, período en que la especie comienza alimentarse, debido a que durante la época de crecida del río, los peces tienen abundante alimento que encuentran en las áreas inundadas (Campos, 2005).

\section{TALLA A LA PRIM ERA M ADUREZSEXUAL}

Potamorhina altamazonica alcanza la talla de primera madurez sexual $\left(\mathrm{L}_{50}\right)$ a los $16,3 \mathrm{~cm}$ de longitud estándar en hembras y $15,5 \mathrm{~cm}$ en machos, siendo las hembras significativamente diferente a los machos (t-Test, $p<0.001)$. Estudios realizado en el lago Catalão de la Amazonía central (Brasil) reporta que la especie en estudio alcanza la $\mathrm{L}_{50}$ a $118 \mathrm{~mm}$ de longitud estándar en hembras (Amadio \& Bittencourt, 2005), menor a la reportada en nuestro estudio. Que probablemente se deba a la procedencia de cuencas donde fueron estudiadas las poblaciones. Nuñez \& Duponchelle (2009), mencionan que es importante realizar estos estudios con individuos que estén dentro de la época de reproducción.

\section{FRECUENCIA DE TALLAS COMO INDICADORES DE SOBREPESCA}

El análisis de la información muestra variaciones entre los porcentajes de ejemplares maduros e inmaduros de un año a otro. Durante el 2005 y 2006 un gran porcentaje entre 51 y $64 \%$ de ejemplares desembarcados estaba por debajo del $\mathrm{L}_{50}$, es decir eran inmaduros capturados antes de que pudieran participar aunque sea una vez en el futuro de las generaciones, con talla promedio de captura de $15.9 \pm 2.3$ y $15.5 \pm 2.2$ por debajo del $\mathrm{L}_{50}$. A partir del 2007 al 2010 entre $58 \mathrm{y}$ $83 \%$ de las capturas estuvo compuesta por ejemplares maduros, es decir que tuvieron la oportunidad de reproducirse al menos una vez antes de ser capturados, incrementándose progresivamente la talla promedio de captura de $16.6 \pm 2.2$ a $18.2 \pm 2.8$ que para esos años supero al $\mathrm{L}_{50}$, asegurando de esta manera una recuperación de sus poblaciones naturales debido al reducido porcentaje de ejemplares inmaduros en las capturas (Figura 4).

\section{RELACIÓN PESO - LONGITUD}

El ajuste de la relación entre el peso y la longitud, resulto en la siguiente ecuación: $\mathrm{Wt}=0.0244 . \mathrm{L}_{\mathrm{t}}^{2.9773}$, con un coeficiente de correlación (r) de 0.933. Se considero un número de 1,516 datos, con longitud y peso mínimo de $8,6 \mathrm{~cm}, 25$ gr y una máxima de 26,6 cm, 460gr.

Por todo lo expuesto se recomienda a los desisores de política, se incluya en el reglamento de Ordenamiento Pesquero como la talla mínima de captura de Potamorhina altamazonica en $16.5 \mathrm{~cm}$ de longitud estándar, con la finalidad de asegurar que los ejemplares capturados tengan la posibilidad de reproducirse por lo menos una vez en su vida. 


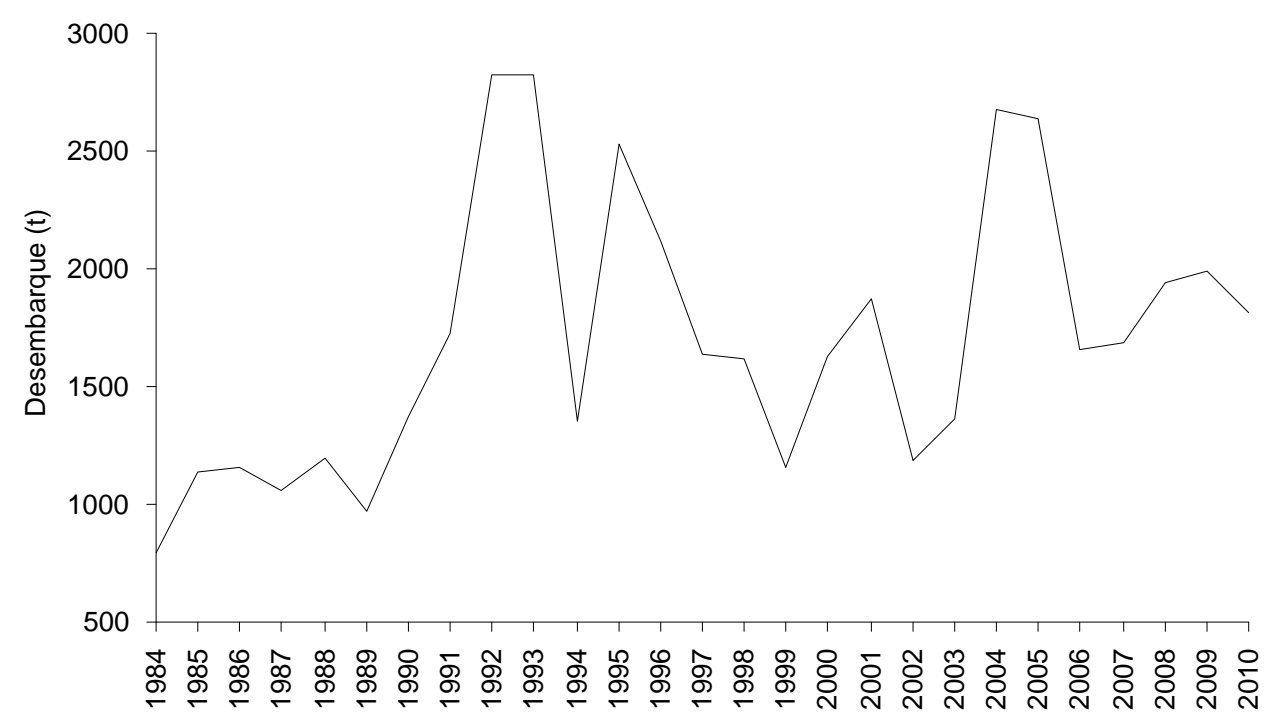

Figura 1. Desembarque total de Potamorhina altamazonica en la región Loreto desde 1984 a 2010.

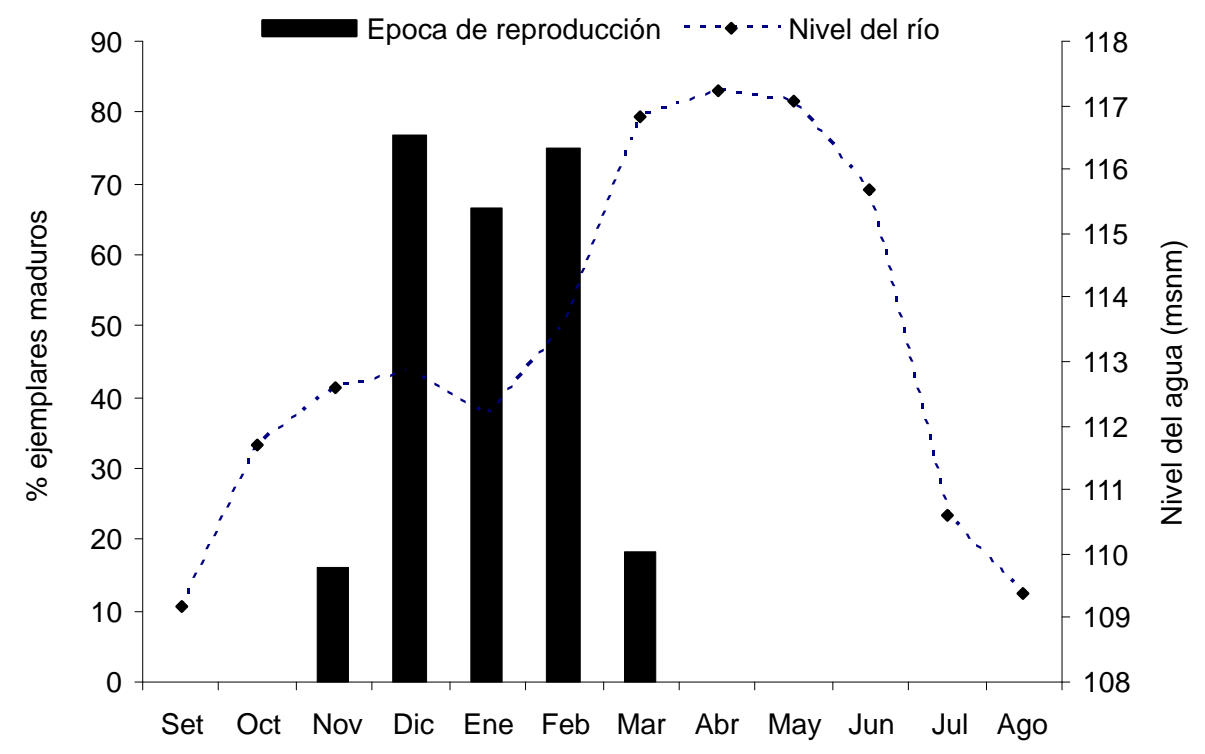

Figura 2. Época de reproducción de Potamorhina altamazonica y su relación con el nivel del agua 


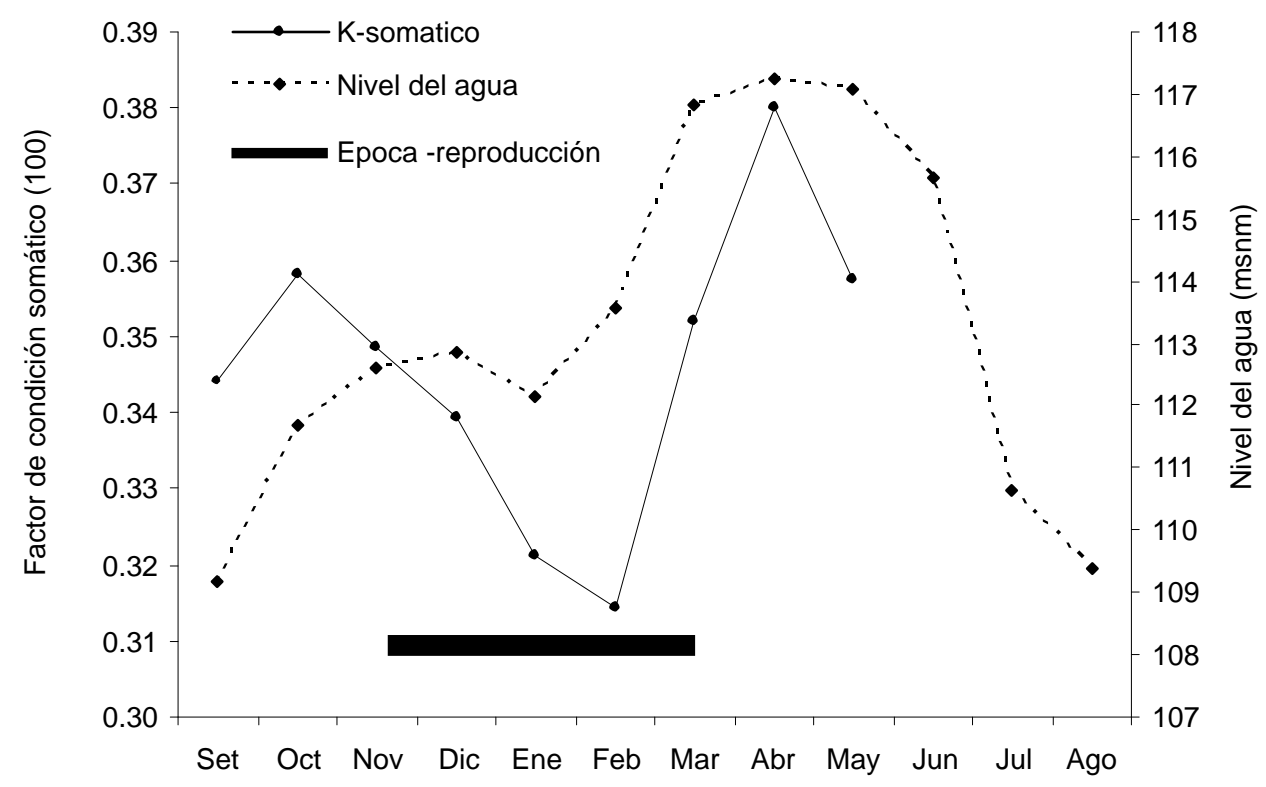

Figura 3. Factor de condición somático de Potamorhina altamazonica y su relación con la época de reproducción.

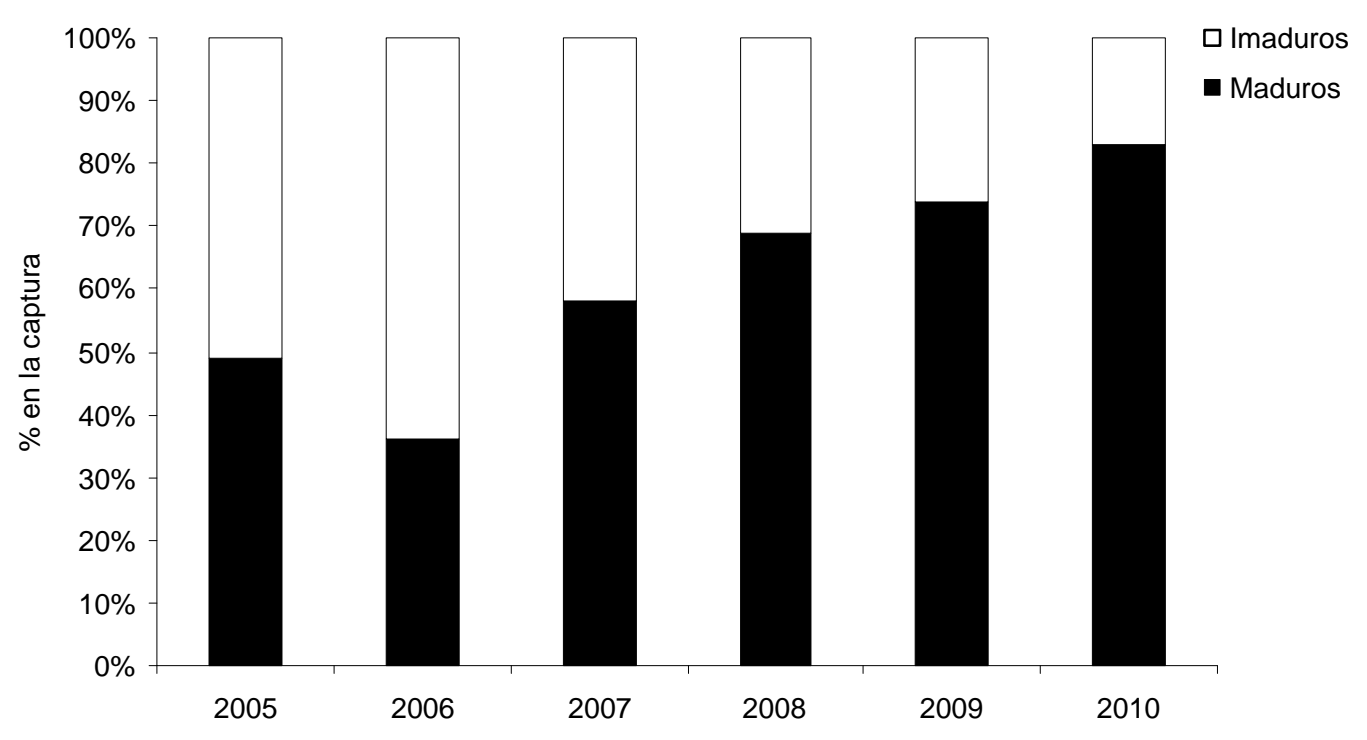

Figura 4. Distribución (\%) de ejemplares maduros e inmaduros de Potamorhina altamazonica en los desembarques entre los años 2005 a 2010. 


\section{BIBLIOGRAFÍA CITADA}

Amadio, A.; Bittencourt, M. 2005. Táticas reprodutivas de peixes em ambientes de várzea na Amaznia Central. Comunicaciones del coloquio internacional sobre Biología de las poblaciones de peces de la Amazonía y piscicultura. 52-59.

Campos, L. 2005. Algunos parámetros físicos, químicos y bioecológicos que influyen en el comportamiento migratorio de la "gamitana" Colossoma macropomum en el río Ucayali. Comunicaciones del coloquio internacional sobre Biología de las poblaciones de peces de la Amazonía y piscicultura. 44-51.

Duponchelle, F.; Cecchi, P.; Corbin, D.; Nuñez, J.; Legendre, M. 1999. Spawning season variations of female Nile tilapia, Oreochromis niloticus, populations from man-made lakes of Côte d'Ivoire. Environmental Biology of Fishes, 56, 377-389.

Duponchelle, F.; Panfili, J. 1998. Variations in age and size at maturity of female Nile tilapia, Oreochromis niloticus, populations from manmade lakes of Côte d'Ivoire. Environmental Biology of Fishes, 52, 453-465.

Froese, R. 2004. Keep it simple: Three indicators to deal with overfishing. Fish. Fisher. 5,86-91.

Froese, R.; Binohlan, C. 2000. Empirical relationships to estimate asymptotic length, length at first maturity and length at maximum yield per recruit in fishes, with a simple method to evaluate length frequency data. J. Fish Biol. 56, 758-773

García, A.; Rodríguez, R.; Montreuil, V. 1996. Longitud de primera maduración y época de desove de dorado Brachyplatystoma flavicans en la Amazonía Peruana. Boletín Científico del INPA. Bogotá, Colombia. N 4:5-17.

García, A.; Tello, S.; Vargas, G.; Duponchelle, F. 2009. Patterns of commercial fish landings in the Loreto region (Peruvian Amazon) between 1984 and 2006. Fish Physiology and Biochemistry 35, 5367.

Goulding, M. 1979. Ecología da Pesca do Río Madeira. INPA. Manaus. Brasil. 172 p

Goulding, M. 1980. The Fishes and the Forest. Explorations in Amazonian Natural History. University of California Press. Berkeley., CA, USA. 280 p.
Isaac, V.; Rocha, V.; Mota, S. 2000. Ciclo reproductivo de algunas especies de peces del medio Amazonas. IBAMA. Serie Estudios de Pesca, 22: 31-64.

Lauzanne, L.; Loubens, G.; Le-Guennec, B. 1990. Pesca y biología pesquera en el Mamoré medio (Región de Trinidad Bolivia). INTERCIENCIA. Vol. 15. $\mathrm{N}^{\mathrm{o}} 6.452-460$.

Lowe-McConnell R.H. 1987. Ecological studies in tropical fish communities. Cambridge, Cambridge University Press. $381 \mathrm{p}$

Nascimento, F. L.; Araujo-Lima, C. 1993. Descrição das larvas de Psectrogaster amazonica e Potamorhina altamazonica (Curimatidae, Pisces) da Amazonía Central. Acta Amazonica 23(4): 457472.

Nuñez, J,; Dunpochelle, F. 2009. Towards a universal scale to assess sexual maturation and related life history traits in oviparous teleost fishes. Fish Physiology and Biochemistry 35: 167-180.

Ruffino, M. L.; Isacc, V. J. 1995. Life cycle and biological parameters of several brazilian amazon fish species. Naga, the ICLARM Quarterly. 41-45.

Smith, N. 1979. A Pesca no Río Amazonas. Instituto Nacional de Pesquisa da Amazonia - Manaus. Brasil. 152 p.

Tello, S.; Montreuil, V.; Maco, J.; Ismiño, R.; Sanchez, H. 1992. Bioecología de peces de importancia económica de la parte inferior de los ríos Ucayali y Marañón. IIAP. Folia Amazónica 4(2): 75-93

Vazzoler, A. E. A. de M.1996. Biologia da reproducao de peixes teleosteos: Teoria e pratica. Universidade Estadual de Maringa Pro-Reitoria de Pesquisa e Pos-Graduacao. Maringa-ParanaBrasil. 169.

Vari, R. P. 1984 (a). Systematics of the Neotropical Characiform Genus Potamorhina (Pisces: Characiformes). Smithsonian Contributios to Zoology, 400: 1-36.

Villacorta, M. 1997. Estudo da idade e crescimento do tambaqui Colossoma macropomun (Characiformes: Characidae) no Amazonas Central, pala análise de marcas sazonais nas estruturas meneralizadas e microestruturas dos otólitos. Tesis de doutorado. Manaus. 215. 